\title{
Rainbow Cycles in Flip Graphs
}

\author{
Stefan Felsner \\ Institut für Mathematik, TU Berlin, Germany \\ felsner@math.tu-berlin.de
}

\section{Linda Kleist}

Institut für Mathematik, TU Berlin, Germany

kleist@math.tu-berlin.de

\section{Torsten Mütze}

Institut für Mathematik, TU Berlin, Germany muetze@math.tu-berlin.de

\section{Leon Sering}

Institut für Mathematik, TU Berlin, Germany sering@math.tu-berlin.de

\begin{abstract}
The flip graph of triangulations has as vertices all triangulations of a convex $n$-gon, and an edge between any two triangulations that differ in exactly one edge. An $r$-rainbow cycle in this graph is a cycle in which every inner edge of the triangulation appears exactly $r$ times. This notion of a rainbow cycle extends in a natural way to other flip graphs. In this paper we investigate the existence of $r$-rainbow cycles for three different flip graphs on classes of geometric objects: the aforementioned flip graph of triangulations of a convex $n$-gon, the flip graph of plane spanning trees on an arbitrary set of $n$ points, and the flip graph of non-crossing perfect matchings on a set of $n$ points in convex position. In addition, we consider two flip graphs on classes of nongeometric objects: the flip graph of permutations of $\{1,2, \ldots, n\}$ and the flip graph of $k$-element subsets of $\{1,2, \ldots, n\}$. In each of the five settings, we prove the existence and non-existence of rainbow cycles for different values of $r, n$ and $k$.
\end{abstract}

2012 ACM Subject Classification Mathematics of computing $\rightarrow$ Combinatorics, Mathematics of computing $\rightarrow$ Permutations and combinations, Mathematics of computing $\rightarrow$ Graph theory, Theory of computation $\rightarrow$ Randomness, geometry and discrete structures

Keywords and phrases flip graph, cycle, rainbow, Gray code, triangulation, spanning tree, matching, permutation, subset, combination

Digital Object Identifier 10.4230/LIPIcs.SoCG.2018.38

Related Version A full version of this paper is available at https://arxiv.org/abs/1712.07421

Acknowledgements We thank Manfred Scheucher for his quick assistance in running computer experiments that helped us to find rainbow cycles in small flip graphs.

\section{Introduction}

Flip graphs are fundamental structures associated with families of geometric objects such as triangulations, plane spanning trees, non-crossing matchings, partitions or dissections. A classical example is the flip graph of triangulations. The vertices of this graph $G_{n}^{\mathrm{T}}$ are the triangulations of a convex $n$-gon, and two triangulations are adjacent whenever they differ by exactly one edge. In other words, moving along an edge of $G_{n}^{\mathrm{T}}$ corresponds to flipping the diagonal of a convex quadrilateral formed by two triangles. Figure 1 shows the graph $G_{6}^{\mathrm{T}}$.

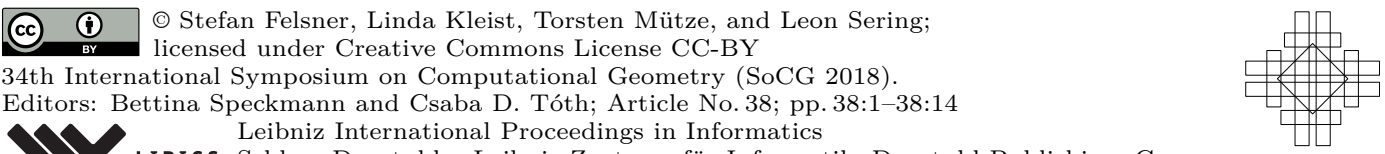




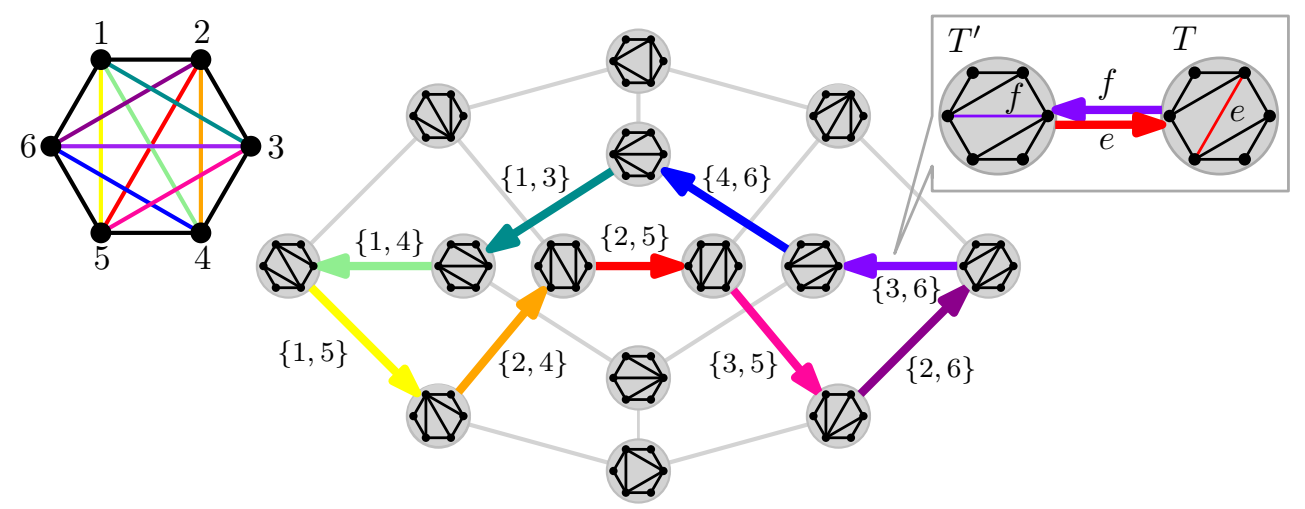

Figure 1 The flip graph of triangulations $G_{6}^{\mathrm{T}}$ with a highlighted rainbow cycle.

A question that has received considerable attention is to determine the diameter of $G_{n}^{\mathrm{T}}$, i.e., the number of flips that is necessary and sufficient to transform any triangulation into any other, see the survey [7]. In a landmark paper [33], Sleator, Tarjan and Thurston proved that the diameter of $G_{n}^{\mathrm{T}}$ is $2 n-10$ for sufficiently large $n$. Recently, Pournin [28] gave a combinatorial proof that the diameter is $2 n-10$ for all $n>12$. A challenging algorithmic problem in this direction is to efficiently compute a minimal sequence of flips that transforms two given triangulations into each other, see [24, 29]. These questions involving the diameter of the flip graph become even harder when the $n$ points are not in convex, but in general position, see e.g. [11, 15, 19]. Moreover, apart from the diameter, many other properties of the flip graph $G_{n}^{\mathrm{T}}$ have been investigated, e.g., its realizability as a convex polytope [9], its automorphism group [23], the vertex-connectivity [18], and the chromatic number [12].

Another property of major interest is the existence of a Hamilton cycle in $G_{n}^{\mathrm{T}}$. This was first established by Lucas [25] and a very nice and concise proof was given by Hurtado and Noy [18]. The reason for the interest in Hamilton cycles is that a Hamilton cycle in $G_{n}^{\mathrm{T}}$ corresponds to a so-called Gray code, i.e., an algorithm that allows to generate each triangulation exactly once, by performing only a single flip operation when moving to the next triangulation. In general, the task of a Gray code algorithm is to generate all objects in a particular combinatorial class, each object exactly once, by applying only a small transformation in each step, such as a flip in a triangulation. Combinatorial classes of interest include geometric configurations such as triangulations, plane spanning trees or non-crossing perfect matchings, but also classes without geometric information such as permutations, combinations, bitstrings etc. This fundamental topic is covered in depth in the most recent volume of Knuth's seminal series The Art of Computer Programming [21], and in the classical books by Nijenhuis and Wilf $[27,36]$. Here are some important Gray code results in the geometric realm: Hernando, Hurtado and Noy [16] proved the existence of a Hamilton cycle in the flip graph of non-crossing perfect matchings on a set of $2 m$ points in convex position for every even $m \geq 4$. Aichholzer et al. [1] described Hamilton cycles in the flip graphs of plane graphs on a general point set, for plane and connected graphs and for plane spanning trees on a general point set. Huemer et al. [17] constructed Hamilton cycles in the flip graphs of non-crossing partitions of a point set in convex position, and for the dissections of a convex polygon by a fixed number of non-crossing diagonals.

As mentioned before, a Hamilton cycle in a flip graph corresponds to a cyclic listing of all objects in some combinatorial class, such that each object is encountered exactly once, by performing a single flip in each step. In this work we consider the dual problem: we are 


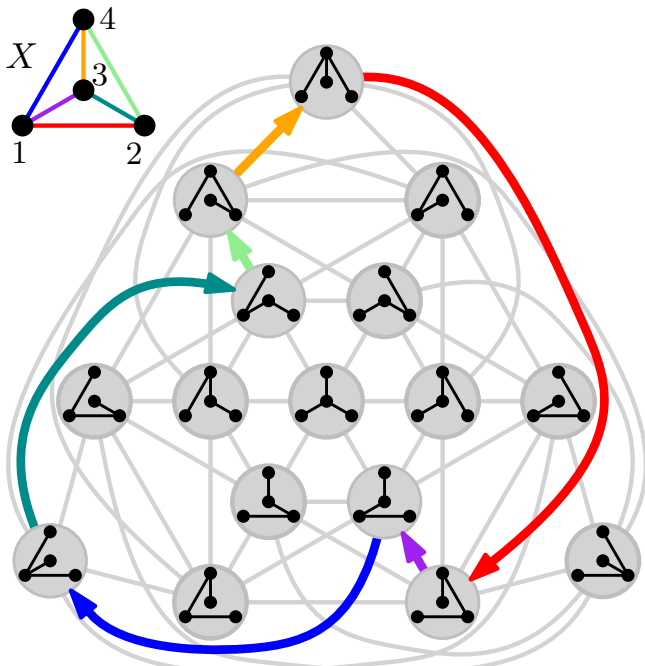

(a) Flip graph of plane spanning trees $G_{X}^{\mathrm{S}}$.

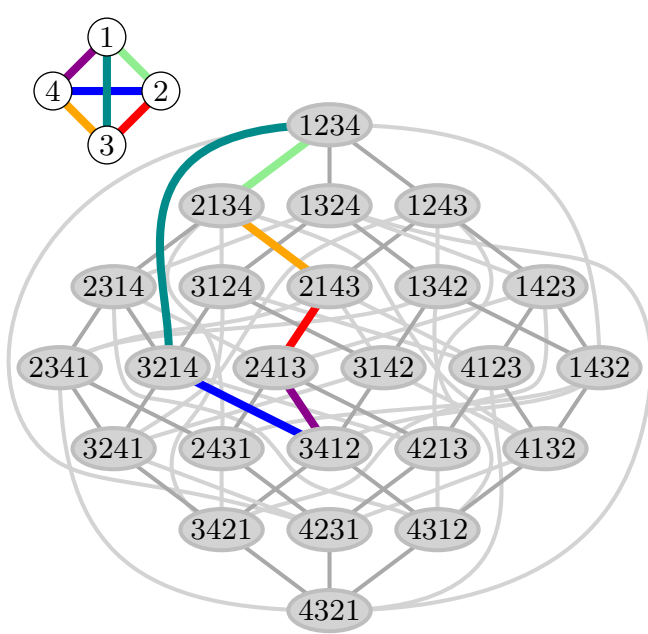

(c) Flip graph of permutations $G_{4}^{\mathrm{P}}$.

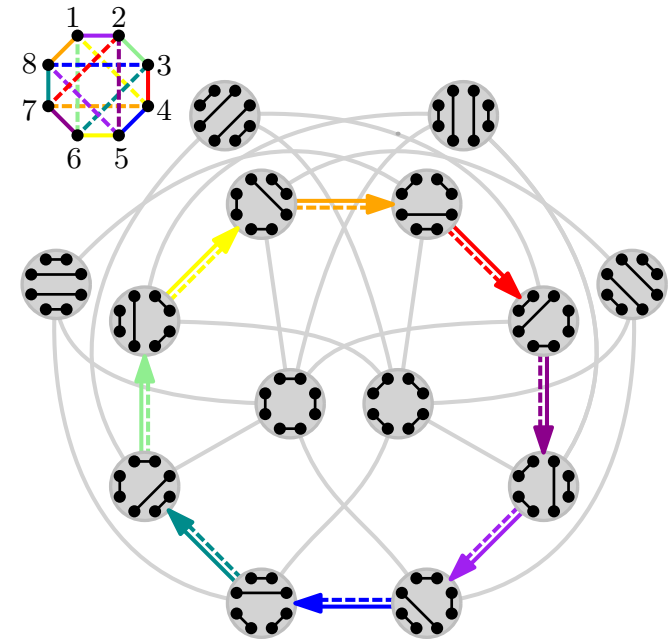

(b) Flip graph of non-crossing perfect matchings $G_{4}^{\mathrm{M}}$.

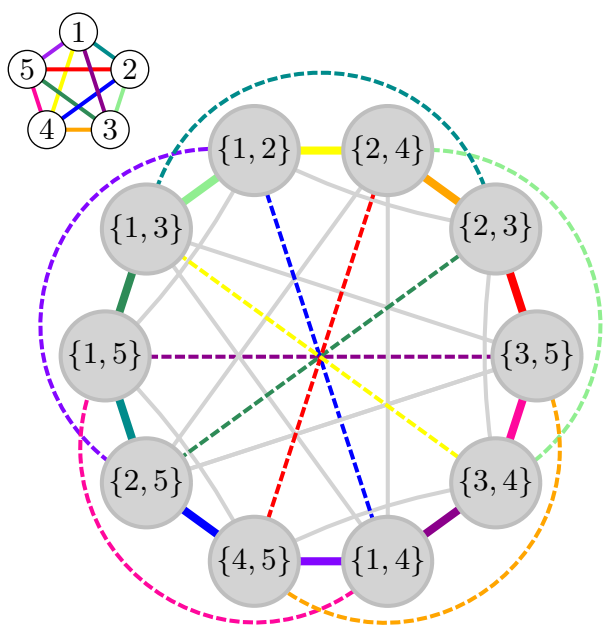

(d) Flip graph of subsets $G_{5,2}^{\mathrm{C}}$.

Figure 2 Examples of flip graphs with 1-rainbow cycles. In (d), two edge-disjoint rainbow Hamilton cycles in $G_{5,2}^{\mathrm{C}}$ are highlighted, one with bold edges and one with dashed edges.

interested in a cyclic enumeration of some of the combinatorial objects, such that each flip operation is encountered exactly once. For instance, in the flip graph of triangulations $G_{n}^{\mathrm{T}}$, we ask for the existence of a cycle with the property that each inner edge of the triangulation appears (and disappears) exactly once. An example of such a cycle is shown in Figure 1. This idea can be formalized as follows. Consider two triangulations $T$ and $T^{\prime}$ that differ in flipping the diagonal of a convex quadrilateral, i.e., $T^{\prime}$ is obtained from $T$ by removing the diagonal $e$ and inserting the other diagonal $f$. We view the edge between $T$ and $T^{\prime}$ in the flip graph $G_{n}^{\mathrm{T}}$ as two arcs in opposite directions, where the arc from $T$ to $T^{\prime}$ receives the label $f$, and the arc from $T^{\prime}$ to $T$ receives the label $e$, so the label corresponds to the edge of the triangulation that enters in this flip; see the right hand side of Figure 1. Interpreting the 
labels as colors, we are thus interested in a directed cycle in the flip graph in which each color appears exactly once, and we refer to such a cycle as a rainbow cycle. More generally, for any integer $r \geq 1$, an $r$-rainbow cycle in $G_{n}^{\mathrm{T}}$ is a cycle in which each edge of the triangulation appears (and disappears) exactly $r$ times. Note that a rainbow cycle does not need to visit all vertices of the flip graph. Clearly, this notion of rainbow cycles extends in a natural way to all the other flip graphs discussed before, see Figure 2.

\subsection{Our results}

In this work we initiate the investigation of rainbow cycles in flip graphs for five popular classes of combinatorial objects. We consider three geometric classes: triangulations of a convex polygon, plane spanning trees on point sets in general position, and non-crossing perfect matchings on point sets in convex position. In addition, we consider two classes without geometric information: permutations of the set $[n]:=\{1,2, \ldots, n\}$, and $k$-element subsets of $[n]$. We proceed to present our results in these five settings in the order they were just mentioned. For the reader's convenience, all results are summarized in Table 1.

Our first result is that the flip graph of triangulations $G_{n}^{\mathrm{T}}$ defined in the introduction has a 1-rainbow cycle for $n \geq 4$ and a 2-rainbow cycle for $n \geq 7$ (Theorem 1 in Section 2).

Next, we consider the flip graph $G_{X}^{\mathrm{S}}$ of plane spanning trees on a point set $X$ in general position; see Figure 2 (a). We prove that $G_{X}^{\mathrm{S}}$ has an $r$-rainbow cycle for any point set $X$ with at least three points for any $r=1,2, \ldots,|X|-2$ (Theorem 2 in Section 3).

We then consider the flip graph $G_{m}^{\mathrm{M}}$ of non-crossing perfect matchings on $2 m$ points in convex position; see Figure 2 (b). We exhibit 1-rainbow cycles for $m=2$ and $m=4$ matching edges, and 2-rainbow cycles for $m=6$ and $m=8$. We argue that there is no 1-rainbow cycle

Table 1 Overview of results.

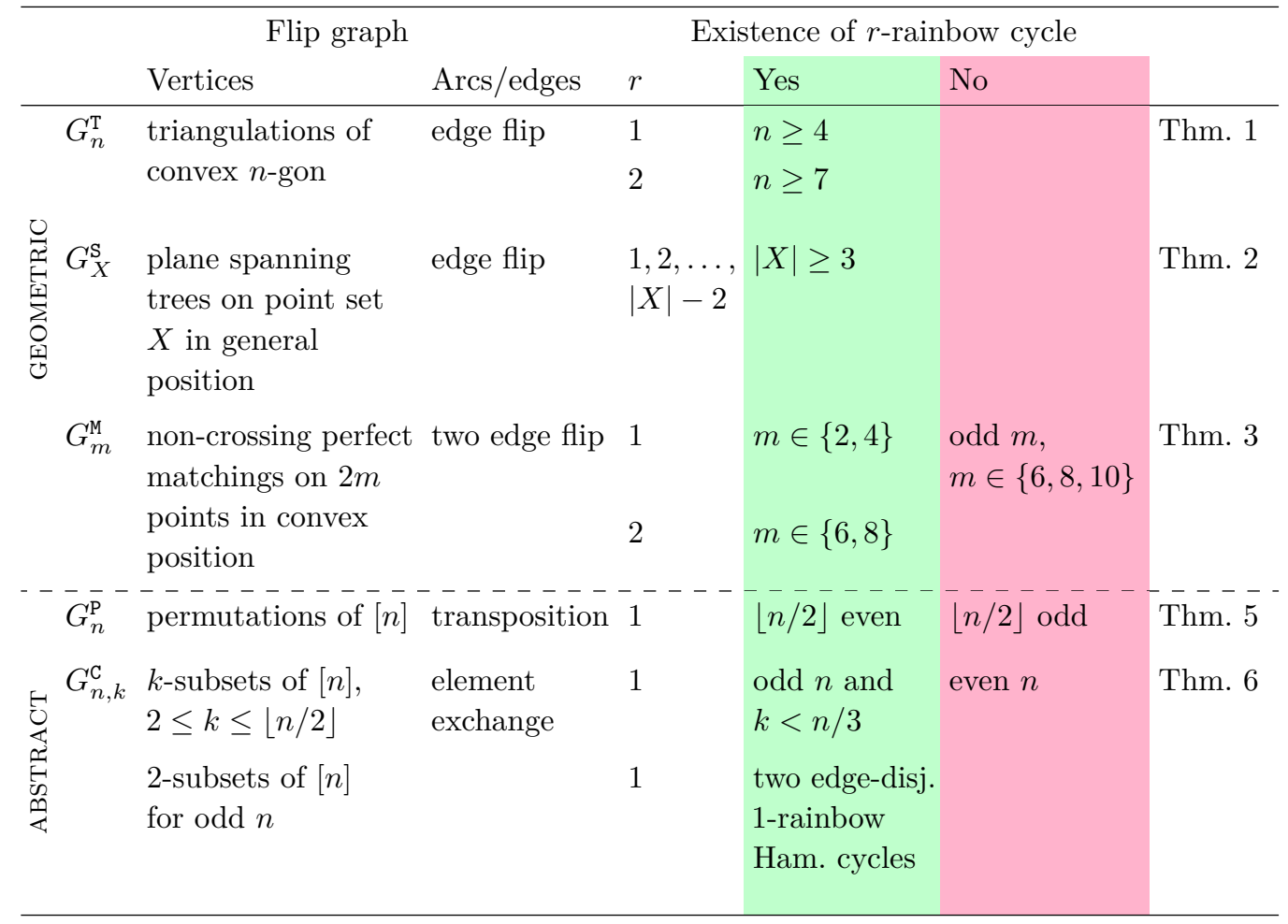


for $m \in\{6,8,10\}$, and none for odd $m$. In fact, we believe that there are no 1-rainbow cycles in $G_{m}^{\mathrm{M}}$ for any $m \geq 5$. Our results for this setting are summarized in Theorem 3 in Section 4 .

Next, we consider the flip graph $G_{n}^{\mathrm{P}}$ of permutations of $[n]$, where an edge connects any two permutations that differ in a transposition, i.e., in exchanging two elements at positions $i$ and $j$; see Figure 2 (c). The edges of this graph are colored with the corresponding pairs $\{i, j\}$, and in a 1-rainbow cycle each of the $\left(\begin{array}{l}n \\ 2\end{array}\right)$ possible pairs appears exactly once. We prove that $G_{n}^{\mathrm{P}}$ has a 1 -rainbow cycle if $\lfloor n / 2\rfloor$ is even, and no 1-rainbow cycle if $\lfloor n / 2\rfloor$ is odd (Theorem 5 in Section 5).

Finally, we consider the flip graph $G_{n, k}^{\mathrm{C}}$ of $k$-element subsets of $[n]$, also known as $(n, k)$ combinations, where an edge connects any two subsets that differ in exchanging one element $i$ for another element $j$; see Figure $2(\mathrm{~d})$. The edges of this graph are colored with the corresponding pairs $\{i, j\}$, and in a 1-rainbow cycle each of the $\left(\begin{array}{l}n \\ 2\end{array}\right)$ possible pairs appears exactly once. Since $G_{n, k}^{\mathrm{C}}$ is isomorphic to $G_{n, n-k}^{\mathrm{C}}$ including the edge-coloring, we assume without loss of generality that $2 \leq k \leq\lfloor n / 2\rfloor$. We prove that $G_{n}^{\mathrm{C}}$ has a 1 -rainbow cycle for every odd $n$ and $k<n / 3$, and we prove that it has no 1-rainbow cycle for any even $n$. The case $k=2$ is of particular interest since a 1 -rainbow cycle in the flip graph $G_{n, 2}^{\mathrm{C}}$ is also a Hamilton cycle. Moreover, we show that $G_{n, 2}^{\mathrm{C}}$ has in fact two edge-disjoint 1-rainbow Hamilton cycles. Our results in this setting are summarized in Theorem 6 in Section 6.

We conclude in Section 7 with some open problems.

\subsection{Related work}

The binary reflected Gray code is a classical algorithm to generate all $2^{n}$ bitstrings of length $n$ by flipping a single bit in each step; see [21, 36]. Since its invention, binary Gray codes satisfying various additional constraints have been constructed; see [32]. Specifically, a Gray code with the property that the bit-flip counts in each of the $n$ coordinates are balanced, i.e., they differ by at most 2, was first described by Tootill [34]; see also [6]. When $n$ is a power of two, every bit appears and disappears exactly $1 / 2 \cdot 2^{n} / n=: r$ many times. This balanced Gray code therefore corresponds to an $r$-rainbow cycle in the corresponding flip graph. In this light, our results are a first step towards balanced Gray codes for other combinatorial classes. For 2-element subsets, we indeed construct perfectly balanced Gray codes.

The Steinhaus-Johnson-Trotter algorithm [20, 35], also known as 'plain changes', is a method to generate all permutations of $[n]$ by adjacent transpositions $i \leftrightarrow i+1$. More generally, it was shown in [22] that all permutations of $[n]$ can be generated by any set of transpositions that form a spanning tree on the set of positions $[n]$; see also [31].

The generation of $(n, k)$-combinations subject to certain restrictions on admissible exchanges $i \leftrightarrow j$ has been studied widely. Specifically, it was shown that all $(n, k)$-combinations can be generated with only allowing exchanges of the form $i \leftrightarrow i+1[8,10,30]$, provided that $n$ is even and $k$ is odd, or $k \in\{0,1, n-1, n\}$. The infamous middle levels conjecture asserts that all $(2 k, k)$-combinations can be generated with only exchanges of the form $1 \leftrightarrow i$, and this conjecture has recently been proved in $[14,26]$.

Rainbow cycles and paths have also been studied in graphs other than flip graphs. A wellknown conjecture in this context due to Andersen [4] asserts that every properly edge-colored complete graph on $n$ vertices has a rainbow path of length $n-2$, i.e., a path that has distinct colors along each of its edges. Progress towards resolving this conjecture was recently made by Alon, Pokrovskiy and Sudakov [2], and Balogh and Molla [5]. 


\subsection{Outline of this paper}

In the remainder of this paper, we present our results discussed before and summarized in Table 1 in the same order. Due to space limitations, we only present particularly illuminating proofs and also provide proof sketches. Full proofs can be found in the preprint version of this paper [13].

\section{Triangulations}

In this section we consider a convex $n$-gon on points labeled clockwise by $1,2, \ldots, n$, and we denote by $\mathcal{T}_{n}$ the set of all triangulations on these points. The graph $G_{n}^{\mathrm{T}}$ has $\mathcal{T}_{n}$ as its vertex set, and an arc $\left(T, T^{\prime}\right)$ between two triangulations $T$ and $T^{\prime}$ that differ in exchanging the diagonal $e \in T$ of a convex quadrilateral formed by two triangles for the other diagonal $f \in T^{\prime}$; see Figure 1. We refer to this operation as a flip, and we denote it by $(e, f)$. Furthermore, we label the arc $\left(T, T^{\prime}\right)$ with the edge $f$, i.e., the edge that enters the triangulation in this flip. The set of arc labels of $G_{n}^{\mathrm{T}}$ is clearly $E_{n}:=\{\{i, j\} \mid j-i>1\} \backslash\{1, n\}$. Recall that we think of these labels as colors. An $r$-rainbow cycle in $G_{n}^{\mathrm{T}}$ is a directed cycle along which every label from $E_{n}$ appears exactly $r$ times. Clearly, the length of an $r$-rainbow cycle equals $r\left|E_{n}\right|=r\left(\left(\begin{array}{l}n \\ 2\end{array}\right)-n\right)$. Given an $r$-rainbow cycle, the cycle obtained by reversing the orientation of all arcs is also an $r$-rainbow cycle since every edge that appears $r$ times also disappears $r$ times. The following theorem summarizes the results of this setting.

- Theorem 1. The flip graph of triangulations $G_{n}^{\mathrm{T}}$ has the following properties:

(i) If $n \geq 4$, then $G_{n}^{\mathrm{T}}$ has a 1-rainbow cycle.

(ii) If $n \geq 7$, then $G_{n}^{\mathrm{T}}$ has a 2-rainbow cycle.

Proof. Let $S_{i}$ be the star triangulation with respect to the point $i$, i.e., the triangulation where the point $i$ has degree $n-1$. To transform $S_{1}$ into $S_{2}$ we can use the flip sequence

$$
F_{n, 1}:=((\{1,3\},\{2,4\}),(\{1,4\},\{2,5\}),(\{1,5\},\{2,6\}), \ldots,(\{1, n-1\},\{2, n\})) .
$$

For any $i=1,2, \ldots, n$, let $F_{n, i}$ denote the flip sequence obtained from $F_{n, 1}$ by adding $i-1$ to all points on the right-hand side of (1). Here and throughout this proof addition is to be understood modulo $n$ with $\{1,2, \ldots, n\}$ as representatives for the residue classes. Note that $F_{n, i}$ transforms $S_{i}$ into $S_{i+1}$ for any $i \in[n]$, and all the edges from $E_{n}$ that are incident with the point $i+1$ appear exactly once during that flip sequence. Note also that $F_{n, i}$ has length $n-3$.

We begin by proving (ii). The concatenation $\left(F_{n, 1}, F_{n, 2}, \ldots, F_{n, n}\right)$ is a flip sequence which applied to $S_{1}$ leads back to $S_{1}$. Along the corresponding cycle $C$ in $G_{n}^{\mathrm{T}}$, every edge from $E_{n}$ appears exactly twice. Specifically, every edge $\{i, j\} \in E_{n}$ appears in the flip sequences $F_{n, i-1}$ and $F_{n, j-1}$. It remains to show that $C$ is indeed a cycle, i.e., every triangulation appears at most once. For this observe that when applying $F_{n, i}$ to $S_{i}$, then for every $j=1,2, \ldots, n-4$, in the $j$-th triangulation we encounter after $S_{i}$, the point $i$ is incident with exactly $n-3-j$ diagonals, the point $i+1$ is incident with exactly $j$ diagonals, while all other points are incident with at most two diagonals. Consequently, if $\min _{1 \leq j \leq n-4}\{n-3-j, j\} \geq 3$, then we can reconstruct uniquely, for any given triangulation encountered along $C$, which flip sequence $F_{n, i}$ it belongs to. This condition is satisfied if $n \geq 8$. For $n=7$ it can be verified directly that $C$ is a 2-rainbow cycle.

It remains to prove (i). We claim that for any $n \geq 4$, applying the flip sequence $X_{n}:=\left(F_{4,3}, F_{5,4}, F_{6,5}, \ldots, F_{n-1, n-2}, F_{n, n-1}, F_{n, n}\right)$ to $S_{1}$ yields a 1 -rainbow cycle in $G_{n}^{\mathrm{T}}$. 


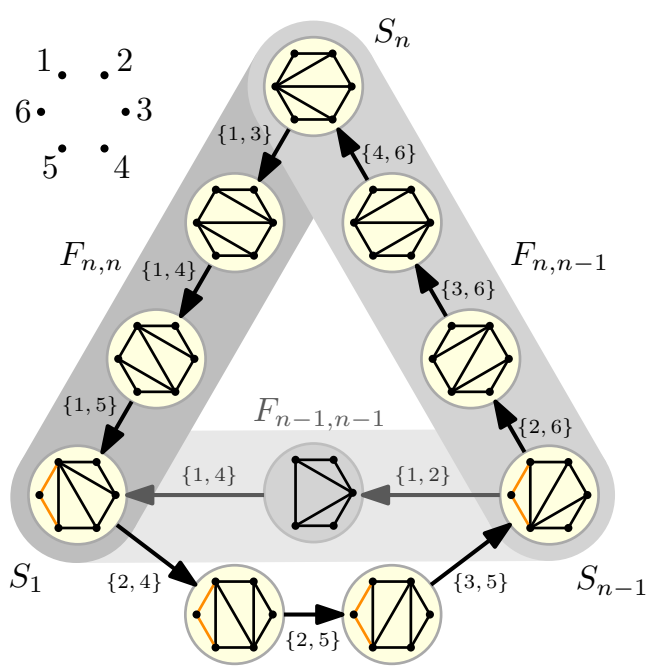

(a) Illustration of the proof of Theorem 1 (i). In the induction step, the rainbow cycle on 5 points is extended to a rainbow cycle on 6 points.

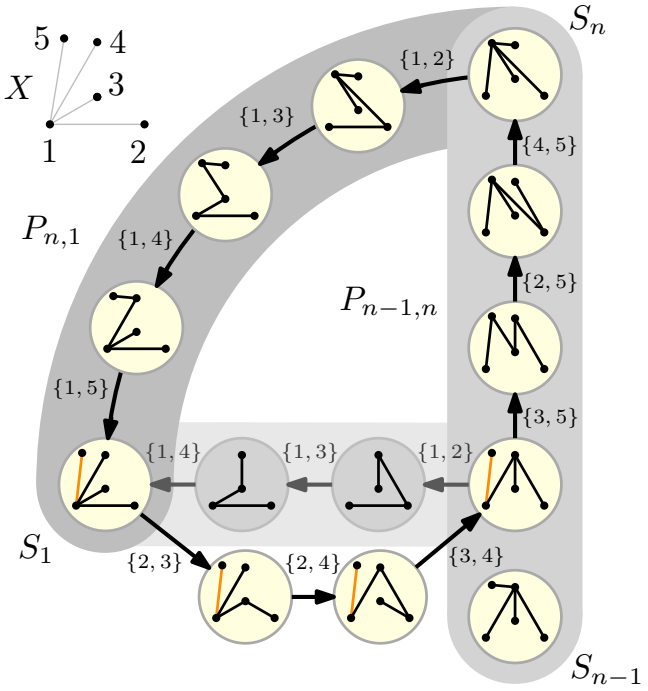

(b) Illustration of the proof of Theorem 2 (i) In the induction step, the rainbow cycle from Figure 2 (a) on the point set [4] is extended to a rainbow cycle on the point set $X=[5]$.

Figure 3

Note that $X_{n}$ differs from $X_{n-1}$ by removing the terminal subsequence $F_{n-1, n-1}$, and by appending $F_{n, n-1}$ and $F_{n, n}$ to the shortened sequence, yielding a sequence of length $\left(\left(\begin{array}{c}n-1 \\ 2\end{array}\right)-(n-1)\right)-(n-4)+2(n-3)=\left(\begin{array}{c}n \\ 2\end{array}\right)-n$; see Figure 3 (a). The fact that $X_{n}$ produces a rainbow cycle follows by induction, by observing that applying $X_{n-1}$ to $S_{1}$ in $G_{n}^{\mathrm{T}}$ yields a cycle along which every edge from $E_{n-1}$ appears exactly once. Moreover, along this cycle the point $n$ is not incident with any diagonals. The modifications described before to construct $X_{n}$ from $X_{n-1}$ shorten this cycle in $G_{n}^{\mathrm{T}}$ and extend it by a detour through triangulations where the point $n$ is incident with at least one diagonal. The set of edges that appear along this cycle is given by $\left(E_{n-1} \backslash E\left(F_{n-1, n-1}\right)\right) \cup E\left(F_{n, n-1}\right) \cup E\left(F_{n, n-1}\right)=E_{n}$, where $E(F)$ denotes the set of edges appearing in a flip sequence $F$. This shows that applying $X_{n}$ to $S_{1}$ yields a 1-rainbow cycle in $G_{n}^{\mathrm{T}}$.

\section{Spanning trees}

In this section we consider plane spanning trees on a set $X$ of $n$ points in general position, i.e., no three points are collinear. The graph $G_{X}^{\mathrm{S}}$ has the vertex set $\mathcal{S}_{X}$ consisting of all plane spanning trees on $X$, and an $\operatorname{arc}\left(T, T^{\prime}\right)$ between two spanning trees $T$ and $T^{\prime}$ that differ in replacing an edge $e \in T$ by another edge $f \in T^{\prime}$; see Figure 2 (a). We denote this flip by $(e, f)$ and label the arc $\left(T, T^{\prime}\right)$ with the edge $f$, i.e., the edge that enters the tree in this flip. Note that the entering edge $f$ alone does not determine the flip uniquely (unlike for triangulations). Clearly, none of the two edges $e$ and $f$ can cross any of the edges in $T \cap T^{\prime}$, but they may cross each other. The set of arc labels of $G_{X}^{\mathrm{S}}$ is clearly $E_{X}:=\left(\begin{array}{c}X \\ 2\end{array}\right)$. An $r$-rainbow cycle in $G_{X}^{\mathrm{S}}$ is a directed cycle along which every label from $E_{X}$ appears exactly $r$ times, so it has length $r\left(\begin{array}{l}n \\ 2\end{array}\right)$. In the following theorem we summarize the results of this setting. 
- Theorem 2. The flip graph of plane spanning trees $G_{X}^{\mathrm{S}}$ has the following properties:

(i) For a point set $X$ with $|X| \geq 3$ in general position, $G_{X}^{\mathrm{S}}$ has a 1-rainbow cycle.

(ii) For a point set $X$ with $|X| \geq 4$ in general position and any $r=2,3, \ldots, m$, where $m:=|X|-1$ if $|X|$ is odd and $m:=|X|-2$ if $|X|$ is even, $G_{X}^{\mathrm{S}}$ has an r-rainbow cycle.

Proof sketch. We first label an arbitrary point on the convex hull of $X$ as point 1, and then label the points from 2 to $n$ in counter-clockwise order around 1 . Moreover, we denote by $S_{i}$ the spanning tree that forms a star with center point $i$. We then define specific flip sequences that transform any star $S_{i}$ into any other star $S_{j}$, yielding paths $P_{i, j}$ in the graph $G_{X}^{\mathrm{s}}$.

For proving (i), we use a similar inductive construction as for the proof of Theorem 1 (i), based on a strengthened induction hypothesis involving the path $P_{n, 1}$; see Figure 3 (b).

To prove (ii) for even $r$, we use a decomposition of $K_{n}$, the complete graph on $n$ vertices, into $r / 2$ Hamilton cycles. Such a decomposition exists by Walecki's theorem [3]. Orienting each of the $r / 2$ Hamilton cycles cyclically, we obtain a directed graph where each vertex has in- and out-degree $r / 2$. We fix an Eulerian cycle $\mathcal{E}$ in this graph, and for each $\operatorname{arc}(i, j) \in \mathcal{E}$ we add the path $P_{i, j}$ from $S_{i}$ to $S_{j}$ to obtain a directed closed tour $C^{\prime}$ in $G_{X}^{\mathrm{S}}$. In this tour every edge $\{i, j\} \in E_{X}$ appears exactly $r$ times, $r / 2$ times in every path towards $S_{i}$ and $r / 2$ times in every path towards $S_{j}$. However, the tour $C^{\prime}$ is not a cycle since every star $S_{i}$ occurs $r / 2$ times. We therefore bypass most of the stars by taking suitable detours. Each detour tree is obtained by exchanging the flip to the star with the flip from the star. This operation transforms $C^{\prime}$ into an $r$-rainbow cycle $C$.

For odd $r$, we perform the previous construction with $(r+1) / 2$ Hamilton cycles, and replace one by the 1-rainbow cycle constructed in part (i).

\section{Matchings}

In this section we consider a set of $n=2 m$ points in convex position labeled clockwise by $1,2, \ldots, n$. Without loss of generality we assume that the points are distributed equidistantly on a circle centered at the origin. $\mathcal{M}_{m}$ denotes the set of all non-crossing perfect matchings with $m$ edges on these points. The graph $G_{m}^{\mathrm{M}}$ has $\mathcal{M}_{m}$ as its vertex set, and an $\operatorname{arc}\left(M, M^{\prime}\right)$ between two matchings $M$ and $M^{\prime}$ that differ in exchanging two edges $e=\{a, b\} \in M$ and $f=\{c, d\} \in M$ for the edges $e^{\prime}=\{a, c\}$ and $f^{\prime}=\{b, d\} \in M^{\prime}$; see Figure $2(\mathrm{~b})$. We refer to this operation as a flip, and label the $\operatorname{arc}\left(M, M^{\prime}\right)$ of $G_{n}^{\mathrm{M}}$ with the edges $e^{\prime}$ and $f^{\prime}$, i.e., the edges that enter the matching in this flip. The set of arc labels of $G_{m}^{\mathrm{M}}$ is $E_{m}:=\{\{i, j\} \mid i, j \in[n]$ and $j-i$ is odd $\}$, and every arc of $G_{m}^{\mathrm{M}}$ carries two such labels. In this definition, the difference $j-i$ must be odd so that an even number of points lies on either side of the edge $\{i, j\}$. An $r$-rainbow cycle in $G_{m}^{\mathrm{M}}$ is a directed cycle along which every label in $E_{m}$ appears exactly $r$ times, two labels in each step, so it has length $r\left|E_{m}\right| / 2=r m^{2} / 2$. The number of vertices of $G_{m}^{\mathrm{M}}$ is the Catalan number $\frac{1}{m+1}\left(\begin{array}{c}2 m \\ m\end{array}\right)$. The following theorem summarizes the results of this setting.

- Theorem 3. The flip graph of non-crossing perfect matchings $G_{m}^{\mathrm{M}}, m \geq 2$, has the following properties:

(i) If $m$ is odd, then $G_{m}^{\mathrm{M}}$ has no 1-rainbow cycle.

(ii) If $m \in\{6,8,10\}$, then $G_{m}^{\mathrm{M}}$ has no 1-rainbow cycle.

(iii) If $m \in\{2,4\}, G_{m}^{\mathrm{M}}$ has a 1-rainbow cycle, and if $m \in\{6,8\}, G_{m}^{\mathrm{M}}$ has a 2-rainbow cycle.

Proof of (i). A 1-rainbow cycle has length $m^{2} / 2$, which is not integral for odd $m$. 

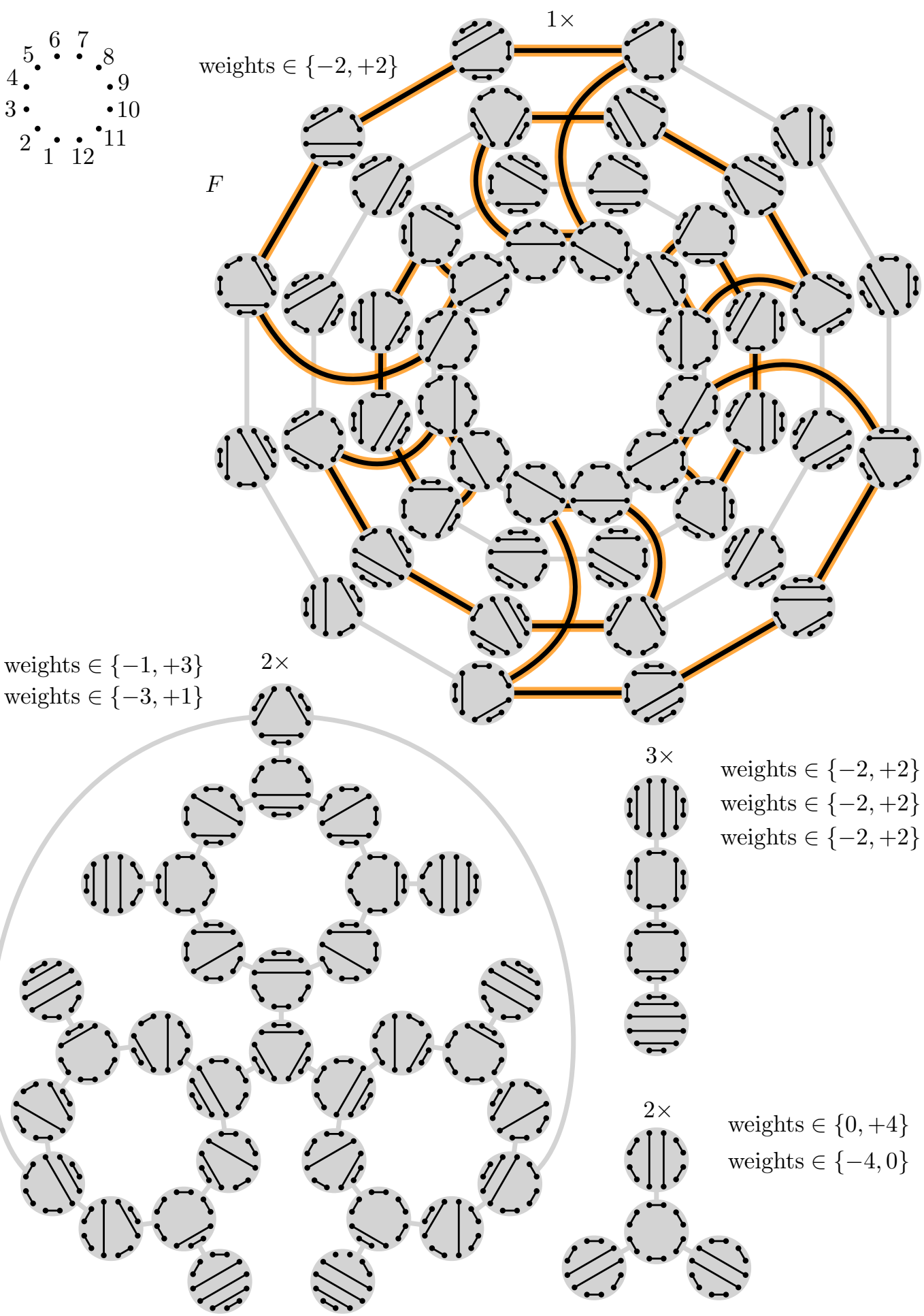

Figure 4 Illustration of the graph $H_{6} \subseteq G_{6}^{\mathrm{M}}$. Some components of this graph are isomorphic to each other and differ only by rotation of the matchings by multiples of $\pi / 6$. Only one representative for each component is shown, together with its multiplicity. The total number of matchings is the 6 th Catalan number 132. The 2-rainbow cycle constructed in the proof of Theorem 3 (iii) is highlighted in the component $F$. 
The length of any edge $e \in E_{m}$, denoted by $\ell(e)$, is the minimum number of points that lie on either of its two sides, divided by two. Consequently, a matching edge on the convex hull has length 0 , whereas the maximum possible length is $(m-2) / 2$, so there are $m / 2$ different edge lengths. A convex quadrilateral formed by four edges from $E_{m}$ is a centered 4-gon, if the sum of the edge lengths of the quadrilateral is $m-2$. Note that this is the maximum possible value. We refer to a flip involving a centered 4-gon as a centered flip. The following observation is crucial for our proof of Theorem 4 (ii).

- Lemma 4. All flips along an r-rainbow cycle in $G_{m}^{\mathrm{M}}$ must be centered flips.

Proof. $E_{m}$ contains exactly $n=2 m$ edges of each length $0,1, \ldots,(m-2) / 2$. Along an $r$-rainbow cycle $C$, exactly $r n$ edges of each length appear and disappear. Consequently, the average length of all edges that appear or disappear along $C$ is $(m-2) / 4$. By definition, in a centered flip the average length of the four edges involved in the flip is exactly the same number; whereas for a non-centered flip, it is strictly smaller. Therefore, $C$ must not contain any non-centered flips.

Lemma 4 allows to restrict our search for rainbow cycles to the subgraph $H_{m}$ of $G_{m}^{\mathrm{M}}$ obtained by considering arcs that represent centered flips; consider Figure 4 for an example.

Proof sketch of (ii). To prove the case $m=6$, we analyze each of the connected components of $H_{6}$ separately. Only one of them contains cycles of length $m^{2} / 2=18$. This component is denoted by $F$ in Figure 4. Analyzing the flip types along the arcs of this component, we show the non-existence of a 1-rainbow cycle in $F$. The proof for the cases $m=8$ and $m=10$ is computer-based, and uses exhaustive search for a 1-rainbow cycle in each connected component of $H_{m}$.

Proof sketch of (iii). There are two non-crossing matchings with $m=2$ edges, connected by two $\operatorname{arcs}$ in $G_{m}^{\mathrm{M}}$ that form 1-rainbow cycle. For $m=4$ a 1-rainbow cycle is shown in Figure 2 (b). A 2-rainbow cycle for $m=6$ is shown in Figure 4. A 2-rainbow cycle for $m=8$ can be constructed using the path $P$ of length 8 between matchings $M$ and $M^{\prime}$ depicted in Figure 5. Note that $M^{\prime}$ differs from $M$ by a counter-clockwise rotation by an angle of $\alpha:=2 \pi / 8$. Repeating this flip sequence eight times, rotating all flips by an angle of $\alpha \cdot i$ for $i=0,1, \ldots, 7$, yields a 2-rainbow cycle in $G_{m}^{\mathrm{M}}$.

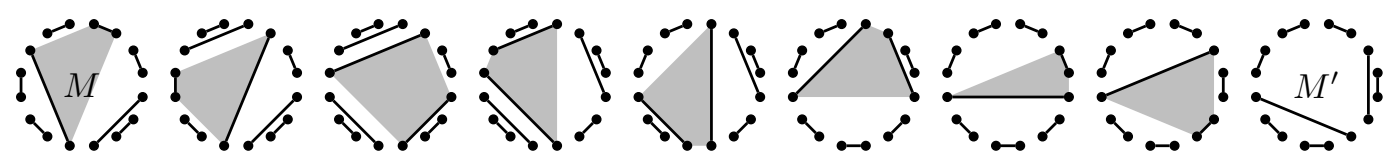

Figure 5 Definition of path $P$ between matchings $M$ and $M^{\prime}$ in $G_{8}^{\mathrm{M}}$.

\section{Permutations}

In this section, we consider the set of permutations $\Pi_{n}$ of $[n]$. The graph $G_{n}^{\mathrm{P}}$ has vertex set $\Pi_{n}$, and an edge $\{\pi, \rho\}$ between any two permutations $\pi$ and $\rho$ that differ in exactly one transposition between the entries at positions $i$ and $j$; see Figure 2 (c). We label the edge $\{\pi, \rho\}$ of $G_{n}^{\mathrm{P}}$ with the transposition $\{i, j\}$. A 1-rainbow cycle in $G_{n}^{\mathrm{P}}$ is a cycle of length $\left(\begin{array}{l}n \\ 2\end{array}\right)$ along which every transposition appears exactly once. The following theorem summarizes the results in this setting. 
- Theorem 5. The flip graph of permutations $G_{n}^{\mathrm{P}}, n \geq 2$, has the following properties:

(i) If $\lfloor n / 2\rfloor$ is odd, then $G_{n}^{\mathrm{P}}$ has no 1-rainbow cycle.

(ii) If $\lfloor n / 2\rfloor$ is even, then $G_{n}^{\mathrm{P}}$ has a 1-rainbow cycle.

Proof sketch. The graph $G_{n}^{\mathrm{P}}$ is bipartite, where the partition classes are given by the parity of the number of inversions. If follows that a cycle of length $\left(\begin{array}{l}n \\ 2\end{array}\right)$ cannot exist when this number is odd, which happens exactly when $\lfloor n / 2\rfloor$ is odd. This proves (i).

To prove (ii), observe that the graph $G_{n}^{\text {p }}$ is vertex-transitive, so it suffices to specify a sequence of $\left(\begin{array}{l}n \\ 2\end{array}\right)$ transpositions that yields a rainbow cycle. We refer to such a sequence as a rainbow sequence for $\Pi_{n}$. For the induction base $n=4$, we use the rainbow sequence $R_{4}:=(\{1,2\},\{3,4\},\{2,3\},\{1,4\},\{2,4\},\{1,3\})$. Applying $R_{4}$ to the permutation 1234 yields the rainbow cycle depicted in Figure 2 (c).

For the induction step, we assume that $n=4 \ell$ for some integer $\ell \geq 1$. From a rainbow sequence $R_{n}$ for $\Pi_{n}$, we construct two new rainbow sequences, one for $\Pi_{n+1}$ and another one for $\Pi_{n+4}$. This covers all values for which $\lfloor n / 2\rfloor$ is even. The inductive construction is based on the following trick: replacing a transposition $\{i, j\}$ in $R_{n}$ by the sequence of three transpositions $(\{i, n+1\},\{i, j\},\{j, n+1\})$ has the same effect on the entries of the permutation, namely, swapping only the entries $i$ and $j$ and leaving the entry $n+1$ at the same position. However, in the modified sequence the transpositions $\{i, n+1\}$ and $\{j, n+1\}$ are used additionally.

\section{Subsets}

In this section we consider the set of all $k$-element subsets of $[n]$, denoted by $C_{n, k}:=\left(\begin{array}{c}{[n]} \\ k\end{array}\right)$, also called $(n, k)$-combinations. The graph $G_{n, k}^{\mathrm{C}}$ has vertex set $C_{n, k}$, and an edge $\{A, B\}$ between two sets $A$ and $B$ that differ in exchanging an element $x$ for another element $y$, i.e., $A \backslash B=\{x\}$ and $B \backslash A=\{y\}$; see Figure $2(\mathrm{~d})$. We label the edge $\{A, B\}$ of $G_{n, k}^{\mathrm{C}}$ with the transposition $A \triangle B=\{x, y\} \in C_{n, 2}$. A 1-rainbow cycle in $G_{n, k}^{\mathrm{c}}$ is a cycle of length $\left(\begin{array}{l}n \\ 2\end{array}\right)$ where every transposition appears exactly once. We only consider 1-rainbow cycles in this setting, and we simply refer to them as rainbow cycles. Note that the number of vertices of $G_{n, k}^{\mathrm{C}}$ is $\left(\begin{array}{l}n \\ k\end{array}\right)$. Consequently, a rainbow cycle for $k=2$ is in fact a Hamilton cycle, i.e., a Gray code in the classical sense. Since $G_{n, k}^{\mathrm{C}}$ and $G_{n, n-k}^{\mathrm{C}}$ are isomorphic, including the edge labels, we may assume without loss of generality that $k \leq n / 2$. Moreover, observe that for $k=1$ and $n>3$, the length of the rainbow cycle exceeds the number of vertices of $G_{n, k}^{\mathrm{C}}$, so we also assume that $k \geq 2$. The following theorem summarizes the results in this setting.

- Theorem 6. Let $n \geq 4$ and $2 \leq k \leq\lfloor n / 2\rfloor$. The flip graph of subsets $G_{n, k}^{\mathrm{C}}$ has the following properties:

(i) If $n$ is even, there is no rainbow cycle in $G_{n, k}^{\mathrm{C}}$.

(ii) If $n$ is odd and $k=2$, then $G_{n, 2}^{\mathrm{C}}$ has a rainbow Hamilton cycle.

(iii) If $n$ is odd and $k=2$, then $G_{n, 2}^{\mathrm{C}}$ has two edge-disjoint rainbow Hamilton cycles.

(iv) If $n$ is odd and $3 \leq k<n / 3$, then $G_{n, k}^{\mathrm{C}}$ has a rainbow cycle.

Proof of Theorem 6 (i). A fixed element $x \in[n]$ is involved in $n-1$ transpositions. If such a transposition is applied to a set containing $x$, then the resulting set does not contain $x$, and vice versa. In a rainbow cycle we return to the starting set and use each of these transpositions exactly once, so $n-1$ must be even, or equivalently, $n$ must be odd.

To prove parts (ii)-(iv) of Theorem 6, we construct rainbow cycles using a rainbow block. To introduce this notion, we need some definitions. For a set $A \subseteq[n], \sigma(A)$ denotes the set 


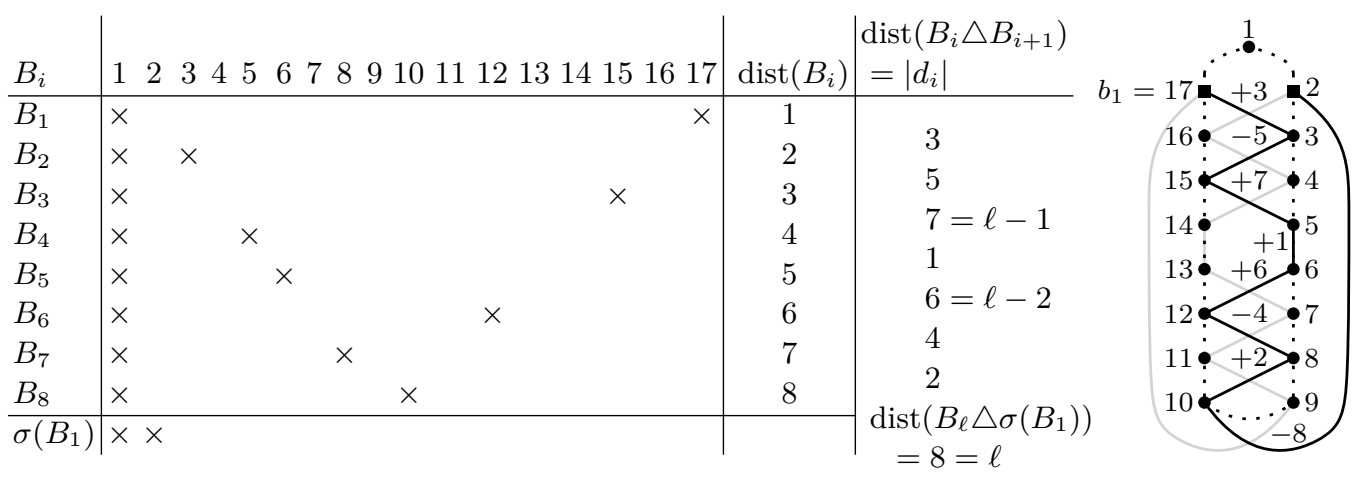

Figure $6 \mathrm{~A}$ rainbow block for $\ell=8$. A cross in row $B_{i}$ and column $j$ indicates that $j \in B_{i}$. On the right, the sequence $\left(b_{1}, b_{2}, \ldots, b_{\ell}, 2\right)$ for this block is depicted as a path drawn in black. The path drawn in gray represents another rainbow block, yielding a rainbow Hamilton cycle that is edge-disjoint from the first cycle.

obtained from $A$ by adding 1 to all elements, modulo $n$ with $\{1,2, \ldots, n\}$ as residue class representatives; for a pair $\{x, y\} \in C_{n, 2}$, we define $\operatorname{dist}(\{x, y\}):=\min \{y-x, x-y\} \in[\ell]$ where the differences are taken modulo $n$. We call a sequence $B=\left(B_{1}, B_{2}, \ldots, B_{\ell}\right)$ with $B_{i} \in C_{n, k}$ a rainbow block if $C(B):=\left(B, \sigma^{1}(B), \sigma^{2}(B), \ldots, \sigma^{2 \ell}(B)\right)$ is a rainbow cycle in $G_{n, k}^{\mathrm{C}}$. By definition, a rainbow cycle built from a rainbow block is highly symmetric.

Proof sketch of Theorem 6 (ii). Let $n=2 \ell+1$ for some integer $\ell \geq 2$. We define a sequence $B=\left(B_{1}, B_{2}, \ldots, B_{\ell}\right)$ of pairs $B_{i} \in C_{n, 2}$ such that the following conditions hold: (a) $B_{i}=\left\{1, b_{i}\right\}$ for $i \in[\ell]$ with $3 \leq b_{i} \leq n$ and $b_{1}=n$, (b) $\left\{\operatorname{dist}\left(B_{i}\right) \mid i \in[\ell]\right\}=[\ell]$, and (c) $\left\{\operatorname{dist}\left(B_{i} \triangle B_{i+1}\right) \mid i \in[\ell-1]\right\} \cup\left\{\operatorname{dist}\left(B_{\ell} \triangle \sigma\left(B_{1}\right)\right\}=[\ell]\right.$. We claim that a sequence $B$ satisfying these conditions yields a rainbow cycle $C=C(B)$. Indeed, (a) ensures that any two consecutive sets in $C$ differ in exactly one transposition, and (b) and (c) guarantee that every pair $A \in C_{n, 2}$ and every transposition $T \in C_{n, 2}$, respectively, appear exactly once along $C$. An example of a rainbow block satisfying these conditions is shown in Figure 6 .

It remains to construct a rainbow block with $B_{i}=\left\{1, b_{i}\right\}$ and $b_{1}=n$. We define

$$
d_{i}:=\left\{\begin{array}{ll}
(-1)^{i+1} \cdot(2 i+1) & \text { if } i \leq\lfloor(\ell-1) / 2\rfloor \\
(-1)^{i} & \text { if } i=\lfloor(\ell+1) / 2\rfloor \\
(-1)^{i+1} \cdot 2(\ell-i) & \text { if } i \geq\lfloor(\ell+2) / 2\rfloor
\end{array} \text { and } b_{i+1}:=b_{i}+d_{i} \bmod n \quad \text { for } i \in[\ell-1] .\right.
$$

This definition satisfies conditions (a) and (b), and $\operatorname{dist}\left(B_{i} \triangle B_{i+1}\right)=\left|d_{i}\right|$ for all $i \in[\ell-1]$. By definition, the set $\left\{\left|d_{i}\right| \mid i \in[\ell-1]\right\}$ equals $[\ell] \backslash\{\ell\}$ if $\ell$ is even and it equals $[\ell] \backslash\{\ell-1\}$ if $\ell$ is odd. These missing numbers are contributed by $\operatorname{dist}\left(B_{\ell} \triangle \sigma\left(B_{1}\right)\right)$, so (c) is satisfied.

The sequence $\left(b_{1}, b_{2}, \ldots, b_{\ell}, 2\right)$ can be interpreted as a path on the vertex set $[n]$; see the right part of Figure 6 . The edge lengths of this path measured along the shorter of the two arcs along the cycle $(1,2, \ldots, n)$ correspond to the $\left|d_{i}\right|$ 's. We use this path representation to prove parts (iii) and (iv) of Theorem 6 .

\section{Open problems}

- For all the combinatorial classes considered in this paper, it would be very interesting to exhibit $r$-rainbow cycles for larger values of $r$, in particular for the flip graphs of permutations and subsets. Another natural next step is to investigate rainbow cycles in 
other flip graphs, e.g., for non-crossing partitions of a convex point set or for dissections of a convex polygon (see [17]).

- We believe that the flip graph of non-crossing perfect matchings $G_{m}^{\mathrm{M}}$ has no 1-rainbow cycle for any $m \geq 5$. This is open for the even values of $m \geq 12$. Moreover, the subgraph $H_{m}$ of $G_{m}^{\mathrm{M}}$ restricted to centered flips (see Figure 4) is a very natural combinatorial object with many interesting properties that deserve further investigation. What is the number of connected components of $H_{m}$ and what is their size? Which components are trees and which components contain cycles?

- We conjecture that the flip graph of subsets $G_{n, k}^{\mathrm{C}}$ has a 1-rainbow cycle for all $2 \leq k \leq n-2$. This is open for $n / 3 \leq k \leq 2 n / 3$. In view of Theorem 6 (iii) we ask: does $G_{n, 2}^{\mathrm{C}}$ have a factorization into $n-2$ edge-disjoint rainbow Hamilton cycles?

\section{References}

1 O. Aichholzer, F. Aurenhammer, C. Huemer, and B. Vogtenhuber. Gray code enumeration of plane straight-line graphs. Graphs Combin., 23(5):467-479, 2007. doi: 10.1007/s00373-007-0750-z.

2 N. Alon, A. Pokrovskiy, and B. Sudakov. Random subgraphs of properly edge-coloured complete graphs and long rainbow cycles. arXiv:1608.07028, Aug 2016.

3 B. Alspach. The wonderful Walecki construction. Bull. Inst. Combin. Appl., 52:7-20, 2008.

4 L. D. Andersen. Hamilton circuits with many colours in properly edge-coloured complete graphs. Mathematica Scandinavica, 64:5-14, 1989.

5 J. Balogh and T. Molla. Long rainbow cycles and Hamiltonian cycles using many colors in properly edge-colored complete graphs. arXiv:1706.04950, Jun 2017.

6 G. S. Bhat and C. D. Savage. Balanced Gray codes. Electron. J. Combin., 3(1):Research Paper 25, approx. 11 pp., 1996. URL: http://www.combinatorics.org/Volume_ 3/Abstracts/v3i1r25.html.

7 P. Bose and F. Hurtado. Flips in planar graphs. Comput. Geom., 42(1):60-80, 2009. doi:10.1016/j. comgeo.2008.04.001.

8 M. Buck and D. Wiedemann. Gray codes with restricted density. Discrete Math., 48(23):163-171, 1984. doi:10.1016/0012-365X (84)90179-1.

9 C. Ceballos, F. Santos, and G. M. Ziegler. Many non-equivalent realizations of the associahedron. Combinatorica, 35(5):513-551, 2015. doi:10.1007/s00493-014-2959-9.

10 P. Eades, M. Hickey, and R. C. Read. Some Hamilton paths and a minimal change algorithm. J. Assoc. Comput. Mach., 31(1):19-29, 1984. doi:10.1145/2422.322413.

11 D. Eppstein. Happy endings for flip graphs. J. Comput. Geom., 1(1):3-28, 2010.

12 R. Fabila-Monroy, D. Flores-Peñaloza, C. Huemer, F. Hurtado, J. Urrutia, and D. R. Wood. On the chromatic number of some flip graphs. Discrete Math. Theor. Comput. Sci., 11(2):47-56, 2009.

13 S. Felsner, L. Kleist, T. Mütze, and L. Sering. Rainbow cycles in flip graphs. arXiv:1712. 07421, Dec 2017.

14 P. Gregor, T. Mütze, and J. Nummenpalo. A short proof of the middle levels theorem. arXiv:1710.08249, Oct 2017.

15 S. Hanke, T. Ottmann, and S. Schuierer. The edge-flipping distance of triangulations. Journal of Universal Computer Science, 2(8):570-579, 1996.

16 C. Hernando, F. Hurtado, and M. Noy. Graphs of non-crossing perfect matchings. Graphs Combin., 18(3):517-532, 2002. doi:10.1007/s003730200038.

17 C. Huemer, F. Hurtado, M. Noy, and E. Omaña-Pulido. Gray codes for non-crossing partitions and dissections of a convex polygon. Discrete Appl. Math., 157(7):1509-1520, 2009. doi:10.1016/j.dam.2008.06.018. 
18 F. Hurtado and M. Noy. Graph of triangulations of a convex polygon and tree of triangulations. Comput. Geom., 13(3):179-188, 1999. doi:10.1016/S0925-7721(99)00016-4.

19 F. Hurtado, M. Noy, and J. Urrutia. Flipping edges in triangulations. Discrete Comput. Geom., 22(3):333-346, 1999. doi:10.1007/PL00009464.

20 S. Johnson. Generation of permutations by adjacent transposition. Math. Comp., 17:282$285,1963$.

21 D. E. Knuth. The art of computer programming. Vol. 4A. Combinatorial algorithms. Part 1. Addison-Wesley, Upper Saddle River, NJ, 2011.

22 V. L. Kompel'makher and V. A. Liskovets. Sequantial generation of arrangements by means of a basis of transpositions. Kibernetica, 3:17-21, 1975.

23 C. W. Lee. The associahedron and triangulations of the $n$-gon. European J. Combin., 10(6):551-560, 1989. doi:10.1016/S0195-6698(89)80072-1.

$24 \mathrm{M}$. Li and L. Zhang. Better approximation of diagonal-flip transformation and rotation transformation. In Computing and Combinatorics, 4th Annual International Conference, COCOON '98, Taipei, Taiwan, R.o.C., August 12-14, 1998, Proceedings, pages 85-94, 1998. doi:10.1007/3-540-68535-9_12.

25 J. M. Lucas. The rotation graph of binary trees is Hamiltonian. J. Algorithms, 8(4):503-535, 1987. doi:10.1016/0196-6774(87)90048-4.

26 T. Mütze. Proof of the middle levels conjecture. Proc. Lond. Math. Soc. (3), 112(4):677-713, 2016. doi:10.1112/plms/pdw004.

27 A. Nijenhuis and H. S. Wilf. Combinatorial algorithms. Academic Press, Inc. [Harcourt Brace Jovanovich, Publishers], New York-London, second edition, 1978. For computers and calculators, Computer Science and Applied Mathematics.

28 L. Pournin. The diameter of associahedra. Adv. Math., 259:13-42, 2014. doi:10.1016/j. aim.2014.02.035.

29 R. O. Rogers. On finding shortest paths in the rotation graph of binary trees. In Proceedings of the Thirtieth Southeastern International Conference on Combinatorics, Graph Theory, and Computing (Boca Raton, FL, 1999), volume 137, pages 77-95, 1999.

30 F. Ruskey. Adjacent interchange generation of combinations. J. Algorithms, 9(2):162-180, 1988. doi:10.1016/0196-6774(88)90036-3.

31 F. Ruskey and C. Savage. Hamilton cycles that extend transposition matchings in Cayley graphs of $S_{n}$. SIAM J. Discrete Math., 6(1):152-166, 1993. doi:10.1137/0406012.

32 C. Savage. A survey of combinatorial Gray codes. SIAM Rev., 39(4):605-629, 1997. doi: 10.1137/S0036144595295272.

33 D. D. Sleator, R. E. Tarjan, and W. P. Thurston. Rotation distance, triangulations, and hyperbolic geometry. J. Amer. Math. Soc., 1(3):647-681, 1988. doi:10.2307/1990951.

34 G. C. Tootill. The use of cyclic permuted codes in relay counting circuits. Proceedings of the IEE - Part B: Radio and Electronic Engineering, 103:432-436, April 1956.

35 H. F. Trotter. Algorithm 115: Perm. Commun. ACM, 5(8):434-435, 1962. doi:10.1145/ 368637.368660 .

36 H. S. Wilf. Combinatorial algorithms: an update, volume 55 of CBMS-NSF Regional Conference Series in Applied Mathematics. Society for Industrial and Applied Mathematics (SIAM), Philadelphia, PA, 1989. doi:10.1137/1.9781611970166. 